MATERIALS RESEARCH SOCIETY

ORGANIZATION, 1979

officers

President

R. J. H. Voorhoeve, Bell Laboratories

Secretary

R. E. Newnham, Penn. State University

Treasurer

S. E. B. Petrie, Kodak

Councillors

Eric Baer, Case Western University

Troy W. Barbee, Stanford University

Geoffrey E. Brock, IBM

Ho Su Chen, Bell Laboratories

Frederick R. Gamble, EXXON

Bill C. Giessen, Northeastern University

K. A. Jackson (Immediate Past President), Bell Laboratories

Frank Lemkey, United Technologies

James W. Mayer, Cal. Tech. University

Mark Myers, Xerox

Helmut Poppa, NASA-AMES

Rustum Roy (Past President), Penn. State University

Lyle Schwartz, Northwestern University

John Silcox, Cornell University

Kathy C. Taylor, General Motors

Jack H. Wernick, Bell Laboratories

Wendell S. Williams, University of Illinois-Urbana

(Newsletter Editor)

\title{
1980 MEETING
}

Next year's meeting will be November 16-21 at the Copley Plaza Hotel in Boston. Program chairmen will be King N. Tu (IBM) and Clyde J. Northrup (Sandia Laboratories). 